\title{
Spatio-Temporal Activity based Tiling for Panorama Streaming
}

\author{
Y.Sanchez \\ Fraunhofer HHI \\ Einsteinufer 37, 10587 \\ Berlin \\ yago.sanchez@ \\ hhi.fraunhofer.de
}

\author{
R. Skupin \\ Fraunhofer HHI \\ Einsteinufer 37, 10587 \\ Berlin \\ robert.skupin@, \\ hhi.fraunhofer.de
}

\author{
C. Hellge \\ Fraunhofer HHI \\ Einsteinufer 37, 10587 \\ Berlin \\ cornelius.hellge@ \\ hhi.fraunhofer.de
}

\author{
T. Schierl \\ Fraunhofer HHI \\ Einsteinufer 37, 10587 \\ Berlin \\ thomas.schierl@, \\ hhi.fraunhofer.de
}

\begin{abstract}
In panorama streaming an arbitrary Region-of-Interest (RoI) of a high-resolution video is transmitted, allowing users to navigate interactively within the videos. Transmitting the whole video becomes unfeasible due to the required high bitrates and sending a single video per user, which is encoded for that specific user (i.e. its RoI), has scalability issues. Tile based panoramic streaming overcomes the mentioned drawbacks by allowing users to receive a set of tiles that match their RoI instead of the whole set of tiles. However, optimal tiling - so that the transmitted bitrate of the RoI content is minimized - is content dependent. In this paper, we propose a model based on a spatio-temporal activity metric so that optimization of the tiling process can be performed in a low complexity manner.
\end{abstract}

\section{KEYWORDS}

Panorama Streaming, Tiling, HEVC, spatio-temporal complexity, Region of Interest

\section{INTRODUCTION}

Panorama streaming services offer the users the possibility of navigating within a high-resolution video, e.g. covering a wide-angle view such as $180^{\circ}$ or $360^{\circ}$. Users indicate an arbitrary Region-of-Interest (RoI) of the video at any time and only this RoI is subsequently transmitted to the user. Figure 1 shows an example of a high-resolution video, where the RoI of the viewer is shown at two different time instants: namely at time $t_{0}$ (left rectangle) and at time $t_{1}$

Permission to make digital or hard copies of all or part of this work for personal or classroom use is granted without fee provided that copies are not made or distributed for profit or commercial advantage and that copies bear this notice and the full citation on the first page. Copyrights for components of this work owned by others than ACM must be honored. Abstracting with credit is permitted. To copy otherwise, or republish, to post on servers or to redistribute to lists, requires prior specific permission and/or a fee. Request permissions from Permissions@acm.org. NOSSDAV'17, June 20-23, 2017, Taipei, Taiwan

(C) 2017 Association for Computing Machinery.ACM ISBN 978-1-4503-5003$7 / 17 / 06 \ldots \$ 15.00$

DOI: http://dx.doi.org/10.1145/3083165.3083176 (right rectangle). In the figure, the spatial plane of the panorama video (i.e. whole panorama video) is shown as well as the two RoIs marked with blue rectangles at the different time instants.

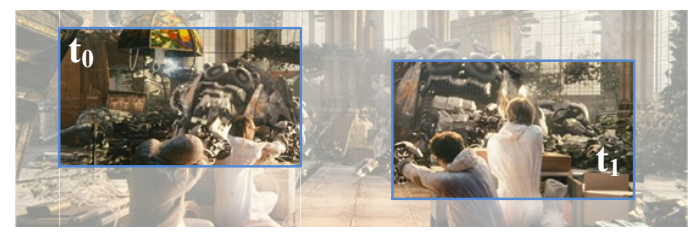

Figure 1: RoI before (t0) and after (t1) user interaction.

There are prototypes and deployed systems already showing panoramic streaming's feasibility [1][2], as well as, commercial products that allow capturing $360^{\circ}$ video in real time [3]. In fact, there are service deployments that offer such functionality to users [4]. Furthermore, the recent market availability of plenty of head mounted displays to consumers such as the Oculus Rift [5], Samsung Gear VR [6] or Google Cardboard [7], is boosting the popularity of interactive panoramic streaming services, which we envision will become ubiquitous in the short term.

Panorama videos typically consist of a very high-spatial resolution signal. Despite the better coding efficiency of new video codecs such as H.265/HEVC, it is still unfeasible to transmit a complete panorama video (e.g. $10 \mathrm{~K} \times 2 \mathrm{~K}$ ) due to the resulting high throughput that would be required. In addition, transmitting the whole panorama at full resolution would waste resources since only a smaller part of it, i.e. the RoI, is used at the receiver side.

The most basic approach to tackle the aforementioned problem consists of encoding and transmitting a per user bitstream that matches the RoI. However, this requires a fully client-server coupled system, where each user indicates its RoI to the server, and this provides a bitstream with the corresponding RoI. Hence, encoding a specific RoI for each single user corresponding to user movements is required. However, having a dedicated encoder per user does not scale well. In order to avoid such issues, tile based panoramic streaming was presented in [8]. The main idea is 
to divide the panorama picture horizontally and vertically into smaller regions that are encoded independently. Then the regions that contain the content belonging to the RoI are transmitted to the user. With this approach, the number of streams does not depend on the number of users but on the number of tiles in which the content has been divided. Therefore, tiled streaming overcomes the scalability issues and allows the server to be stateless and trajectory agnostic, i.e. a plain HTTP server would suffice.

Recently, tile based streaming has become a very popular topic within the research community and standardization bodies. In fact, MPEG has standardized a solution for Dynamic Adaptive Streaming over HTTP (DASH) that allows for tiled streaming (see [9]). Furthermore, many papers have been published in the last years that focus on optimization of tiles [10] or on video delivery of panorama video using tiles [11].

However, one issue that to our knowledge has not yet been properly tackled is how to dimension the tiles in realtime so that the sizes are optimally chosen for a given video. More specifically, how to derive from the video content the optimal tile dimension in a low complexity manner. In [10] or [11], a brute force approach is presented, where all tile dimensions are encoded and the optimum one is selected, which has a high computational complexity. In this paper, we propose a solution based on a pixel domain analysis of the videos, to derive a model using spatio-temporal activity metrics that allows for performing an optimization of the tiling of the videos for panorama streaming, without requiring encoding them at all possible sizes. Therefore, the solution presented in this paper is of a low computational complexity compared to the complexity of the solution requiring encoding the content with different tile sizes.

The remainder of the paper is organized as follows. Section 2 provides an overview of the related work. Section 3 describes the solutions within this paper and Section 4 provides the results and validation of the proposed solution. Finally, Section 5 provides the conclusion of this work.

\section{RELATED WORK}

The work presented here is based on [10]. In [10] the authors discuss on the optimal tile sizes and derive a model based on pixel overhead $\eta$ and bitrate per pixel $\varphi$. When using tile base streaming, a given number of tiles will be transmitted to the user to allow for decoding the user RoI. However, since tiles and RoI do not necessarily coincide exactly, the transmitted video will include additional pixels that will not be presented to the user and lead to a pixel overhead which grows with the size of the tiles. On the other side the lager the tiles are, the be better the coding efficiency of the coded panorama video will be. The transmitted bitrate can be modeled as a function of the RoI size in pixels, the pixel overhead for a given tile size (tile width $t_{w}$ and tile height $t_{h}$ ) and the bitrate per pixel for that given tile size:

$$
B R\left(t_{w}, t_{h}\right)=R_{\text {size }} * \eta\left(t_{w}, t_{h}\right) * \varphi\left(t_{w}, t_{h}\right)
$$

with $\eta$ being:

$$
\eta\left(t_{w}, t_{h}\right)=\frac{\left(r_{w}+t_{w}-1\right) *\left(r_{h}+t_{h}-1\right)}{\operatorname{RoI}_{\text {size }}}
$$

where $r_{w}$ is the RoI width and $r_{h}$ the RoI height. For more information on how to derive the pixel overhead the reader is referred to [10]. Then, the optimization consists of finding the tile sizes that minimizes the transmitted bitrate (BR).

One of the main issues is that, contrary to $\eta$, the bitrate per pixel $\varphi$ is content dependent. One option would be to encode some frames at the beginning of the video at different combinations of $\left(t_{w}, t_{h}\right)$ and use the measured values for the rest of the video. However, covering a satisfying parameter range comes at the cost of an undesirable computational complexity due to the encoding operations. It would be desirable to allow modelling $\varphi$ with a simple analysis of the videos so that no encoding operations are required.

There has been some work in the past that model the influence of the Quantization Parameter (QP) or framerate on video bitrate (see [12] or [13]) based on video characteristics. The idea of the presented work is to derive such a model from spatio-temporal activity metrics. We use the spatio-temporal activity measurements [12], spatial activity (SA) and temporal activity (TA) as follows, since they have been proved to be very valuable for bitrate estimation.

$$
\begin{aligned}
& S A=\operatorname{mean}\left\{\operatorname{std}\left(\operatorname{Sobel}\left[F_{n}\right]\right)\right\} \\
& T A=\operatorname{mean}\left\{\operatorname{std}\left(\left[F_{n}-F_{n-1}\right]\right)\right\} \\
& \text { where } \mathrm{F}_{\mathrm{n}} \text { is the frame number } \mathrm{n} .
\end{aligned}
$$

By trying different functions based on SA and TA, correlation of the function used for estimating the bitrate with the actual values is computed and the one with the highest one is chosen. For more information on the performance of the estimation of the bitrate of a sequence for different QP or framerate values the reader is referred to [13].

\section{PROPOSED SOLUTION}

In this section, we present how to use the spatio-temporal activity measurements for tile optimization. Our focus lies on the optimization of the tiles sizes for an adaptive 
streaming scenario, where several bitrates are offered for adaptation. The bitrate per pixel as used in [10] and shown in Eq. (1) depends on encoding parameters such as QP and applies only for a single operation point, but our aim is to provide the most efficient solution for an adaptive streaming, i.e. for several operation points. Therefore, we reformulate the minimization problem from (1) into minimizing the BD-rate penalty relative to that of the nontiled full panorama encoding. Thus, substitute the term $\varphi$ by the respective $\mathrm{BD}$-rate as follows,

$$
\left(t_{w}^{o p t}, t_{h}^{o p t}\right)=\underset{\left(t_{w}, t_{h}\right)}{\arg } \min \eta\left(t_{w}, t_{h}\right) *\left(1+B D\left(t_{w}, t_{h}\right)\right)(5)
$$

As already mentioned in the previous section, the pixel overhead is easy to be computed and does not depend on the content. We are interested into modeling the $\mathrm{BD}$ rate for different tile sizes. Figure 2 shows the BD-rate of 5 different sequences over different tile sizes (smaller as the number of tiles increases) relative to the non-tiled encoding. A strong dependence of BD-rate on sequence can be seen from Figure 2. For a high number of tiles (very small sizes of $256 \times 128$ pixels) the BD-rate can vary from around $20 \%$ to around $120 \%$.

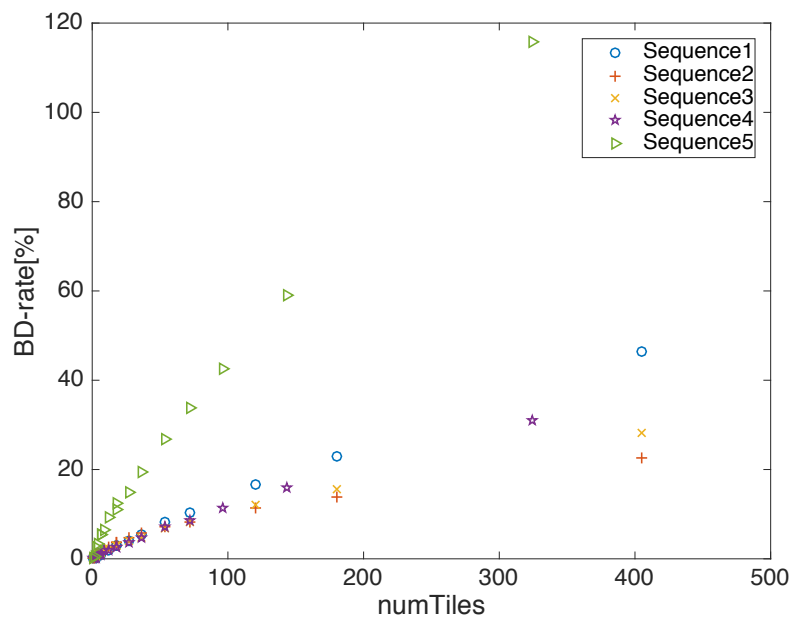

Figure 2: BD-rate over number of tiles.

The 5 sequences used in Figure 2 are the test set used in this work to derive the BD-rate model for the different tile sizes. They have been divided into several tiles as described in Table 1 and encoded following the common test conditions used by JCT-VC during standardization.

The resolution of the first 3 sequences in the test set is equal to $6912 \times 1920$ and the resolution of the last two equal $6912 \times 1536$. All sequences consist of 600 frames and have a framerate of $30 \mathrm{fps}$. Table 1 shows the tiles sizes in which the two different resolutions have been encoded, and indicates the number of tiles that these tile sizes result into. The tiling configurations were chosen to accommodate for a maximum HEVC CTB size of 64x64 pixel.

Table 1: Number of tiles and corresponding tile sizes.

\begin{tabular}{|l|c|c|}
\hline Num. tiles & $\begin{array}{c}\text { Tile sizes for } \\
6912 \times 1920\end{array}$ & $\begin{array}{c}\text { Tile sizes for } \\
6912 \times 1532\end{array}$ \\
\hline 2 & $3456 \times 1920$ & $3456 \times 1536$ \\
\hline 4 & $3456 \times 960$ & $3456 \times 768$ \\
\hline 3 & $2304 \times 1920$ & $2304 \times 1536$ \\
\hline 6 & $2304 \times 960$ & $2304 \times 768$ \\
\hline 9 & $2304 \times 640$ & $2304 \times 512$ \\
\hline 6 & $1152 \times 1920$ & $1152 \times 1536$ \\
\hline 12 & $1152 \times 960$ & $1152 \times 768$ \\
\hline 18 & $1152 \times 640$ & $1152 \times 512$ \\
\hline 18 & $768 \times 960$ & $768 \times 768$ \\
\hline 27 & $768 \times 640$ & $768 \times 512$ \\
\hline 54 & $768 \times 320$ & $768 \times 256$ \\
\hline 36 & $576 \times 640$ & $576 \times 512$ \\
\hline 72 & $576 \times 320$ & $576 \times 256$ \\
\hline $120 / 96^{*}$ & $576 \times 192$ & $576 \times 192$ \\
\hline $180 / 144^{*}$ & $384 \times 192$ & $384 \times 192$ \\
\hline $405 / 314^{*}$ & $256 \times 128$ & $256 \times 128$ \\
\hline
\end{tabular}

*different number of tiles for the different resolution videos

Based on the values shown in Figure 2, we can derive the BD-rate as a function of number of tiles. As can be seen, the BD-rate follows an exponential function with an exponent smaller than 1. Thus, it can be modelled as:

$$
B D_{\text {model }}\left(t_{w}, t_{h}\right)=a *\left(N_{\text {tiles }}\left(t_{w}, t_{h}\right)-1\right)^{b}
$$

where $N_{\text {tiles }}\left(t_{w}, t_{h}\right)=\frac{W_{\text {pano }}}{t_{w}} * \frac{H_{\text {pano }}}{t_{h}}$ and where $\mathrm{W}_{\text {pano }}$ and $\mathrm{H}_{\text {pano }}$ are the width and height of the whole panorama respectively. Note that when the panorama is encoded without tiles (i.e. $\mathrm{N}_{\text {tiles }}=1$ ) the BD-rate is $0 \%$.

Subsequently, we compute the SA, TA of the test sequences that are used to derive a model for $a$ and $b$ parameters that best match the measured BD-rates. The spatio-temporal activity values for the 5 sequences are summarized in Table 2.

Table 2: Spatio-temporal activity values of 5 sequences used to derive the model for the BD-rate

\begin{tabular}{|c|c|c|c|c|c|}
\cline { 2 - 6 } \multicolumn{1}{c|}{} & Seq1 & Seq2 & Seq3 & Seq4 & Seq5 \\
\hline SA & 38.06 & 39.02 & 36.67 & 54.28 & 21.49 \\
\hline TA & 6.34 & 36.66 & 14.08 & 9.67 & 6.08 \\
\hline
\end{tabular}


In order to derive the model for a and $b$, we empirically found two functions for a(SA,TA) and $b(\mathrm{SA}, \mathrm{TA})$ that approximate the curves shown in Figure 2 to a satisfying degree as follows:

$$
\begin{aligned}
& a(S A, T A)=\frac{1}{x_{1}+x_{2} * S A^{x_{3} * T A^{x_{4}}}} \\
& b(S A, T A)=\frac{1}{x_{5}+x_{6} * S A^{x_{7} * T A^{x_{8}}}}
\end{aligned}
$$

Then, in an iterative process we have searched for the values of $\mathrm{x}_{1}-\mathrm{x}_{8}$ that minimize the following error $\varepsilon$ :

$$
\varepsilon=\sum_{\left(t_{w}, t_{h}\right)}\left(\frac{B D_{\text {model }}\left(t_{w}, t_{h}\right)-B D\left(t_{w}^{\min }, t_{h}^{\min }\right)}{B D\left(t_{w}^{\min }, t_{h}^{\min }\right)}\right)^{2}
$$

Therefore, we can write Eq. 6 as Eq. 10:

$$
\begin{aligned}
& B D_{\text {model }}\left(t_{w}, t_{h}\right)= \\
& \quad=\frac{1}{0.82+1.5 * S A^{-0.05} * T A^{-0.04}} *\left(N_{\text {tiles }}\left(t_{w}, t_{h}\right)-1\right)^{\frac{1}{0.32 * S A^{0.33 * T A^{0.11}}}}
\end{aligned}
$$

Figure 3, shows plots of the presented model for each sequence as well as the actual BD-rate values.

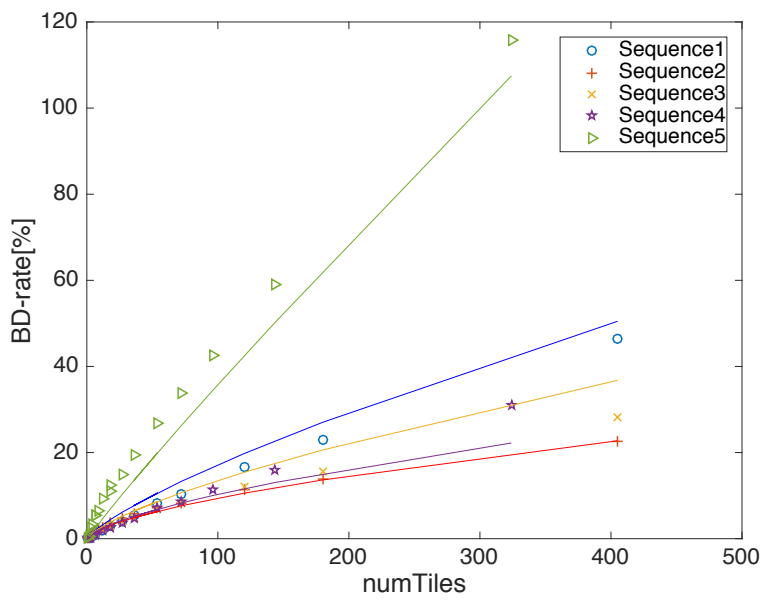

Figure 3: BD-rate model for training sequences.

Finally, with the BD-rate model given in Eq. 10, we can perform the optimization in Eq. 5.

\section{VALIDATION AND RESULTS}

We have performed the optimization as described in Eq. 5 for 4 additional sequences that have not been used to derive the model described in Section 3. Table 3 shows the spatio-temporal activity of the 4 additional sequences.
Table 3: Spatio-temporal activity values of the 4 sequences.

\begin{tabular}{|c|c|c|c|c|}
\cline { 2 - 5 } \multicolumn{1}{c|}{} & Seq6 & Seq7 & Seq8 & Seq9 \\
\hline SA & 40.96 & 33.27 & 28.11 & 37.18 \\
\hline TA & 7.20 & 6.82 & 8.65 & 9.24 \\
\hline
\end{tabular}

The following Figure 4 shows plots of the BD-rate and BD-rate model $_{\text {of }} 4$ sequences:

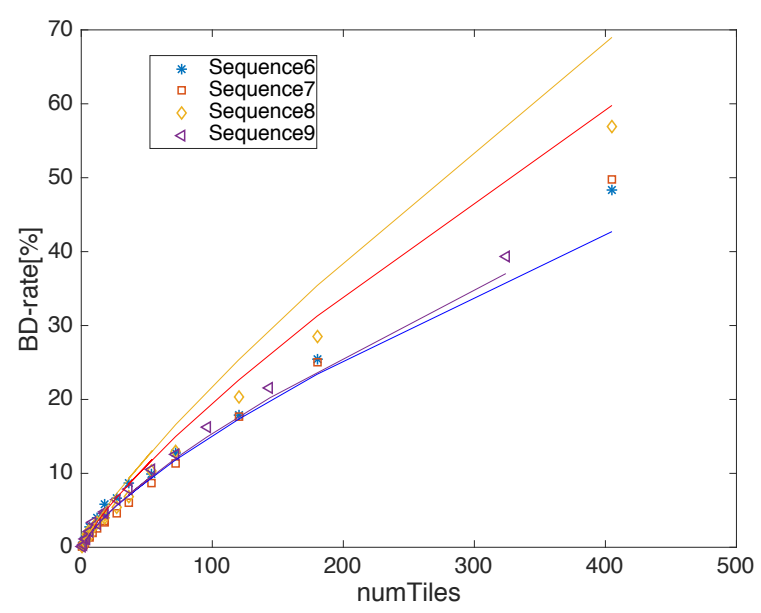

Figure 4: BD-rate model for testing sequences.

As for the test sequences used to derive the BD-rate model, we can see that the model performs desirably for some sequences, e.g. sequence 9, in providing a good approximation of the achievable coding efficiency. On the other hand, comparatively larger errors with respect to BRrate occur for some sequences, e.g. sequence 8 .

However, evaluating model performance in the intended application domain, i.e. tiling granularity estimation, the model precision appears sufficient as explained in the following. We evaluate the performance of the optimization in Eq. 5 when using the model described within the paper in comparison to the actual BD-rate values measured for tiled encodings. Thus, we can evaluate whether irrespective of the model error, the model performs desirably for optimizing the tile sizes.

As shown in Table 4. The result obtained for most of the sequences is the same for using the actual BD-rate with respect to the result when using the model described in Eq. 10. Only for sequence 6 the sizes derived from the optimization differ when using the real BD-rate and the proposed model. 
Table 4: Optimal size using the real BD-rate and the model in Eq. 10

\begin{tabular}{|l|l|l|}
\cline { 2 - 3 } \multicolumn{1}{c|}{} & Real & Model \\
\hline Seq1 & $384 \times 192$ & $384 \times 192$ \\
\hline Seq2 & $256 \times 128$ & $256 \times 128$ \\
\hline Seq3 & $256 \times 128$ & $256 \times 128$ \\
\hline Seq4 & $256 \times 128$ & $256 \times 128$ \\
\hline Seq5 & $576 \times 192$ & $576 \times 192$ \\
\hline Seq6 & $384 \times 192$ & $256 \times 128 *$ \\
\hline Seq7 & $384 \times 192$ & $384 \times 192$ \\
\hline Seq8 & $384 \times 192$ & $384 \times 192$ \\
\hline Seq9 & $256 \times 128$ & $256 \times 128$ \\
\hline
\end{tabular}

*different result when using the model

Finally, we evaluate the achievable BD rates savings of performing the proposed optimization in comparison to taking the most common tile sizes for a RoI of 720p. As seen in Table 4, the most occurring tile sizes among the 9 sequences is $384 \times 192$ for 4 sequences and $256 \times 128$ for other 4 sequences. We compute the BD-rate savings in comparison to having a static configuration, i.e. always $384 \times 192$ or $256 \times 128$ for all sequences following the equation below:

$$
B D-\operatorname{rate}\left(t_{w}, t_{h}\right)=\frac{\eta\left(t_{w}, t_{h}\right) *\left(1+B D\left(t_{w}, t_{h}\right)\right)}{\eta\left(t_{w}, t_{h}\right) *\left(1+B D\left(t_{w}^{s t}, t_{h}^{s t}\right)\right)}
$$

where $t_{w}^{s t}, t_{h}^{s t}$ are the static tile width and height respectively.

Figure 5 shows the BD-rate of the optimal tile sizes in comparison to the static configuration of $256 \times 128$ for the sequences that have another optimal resolution according to the model. We see that the proposed method provides gains from around $2 \%$ up to $14 \%$ BD-rate savings for these sequences. On average gains of $5.9 \%$ are achieved for the sequences in Figure 5.

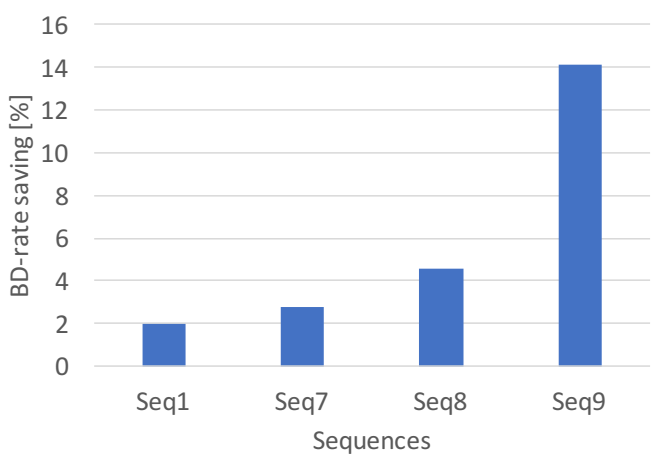

Figure 5: BD-rate savings in comparison to static configuration with tiles of sizes $256 \times 128$.
Figure 6 shows the above comparison for tile size $384 \times 192$ respectively. We see that the proposed method has $1.51 \%$ loss for sequence 2 . The loss can be expected since the used model did not perfectly approximate the coding performance. For sequence 4 the gains are marginal, i.e. of about $0.1 \%$. However, for the rest of the sequences gains from around $1.6 \%$ up to $7 \%$ are obtained. On average gains of $2.5 \%$ are achieved for the sequences in Figure 6.

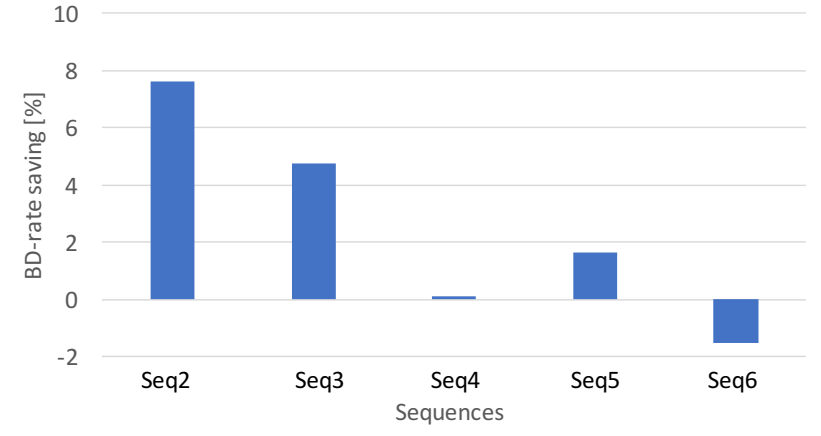

Figure 6: BD-rate savings in comparison to static configuration with tiles of sizes $384 \times 192$.

\section{CONCLUSION}

Tile based panoramic streaming allows users to receive a set of tiles that match their RoI instead of receiving the whole panorama video. However, optimal tiling is content dependent and an optimization process needs to be performed in order to maximize the rate distortion of the transmitted video. In this work, we proposed an optimization approach to achieve this goal. We do that in conjunction with a model to calculate the BD-rate penalty of tiling based on spatio-temporal activity metrics, which has a low complexity compared to performing encodings for each tiling variant in order to measure the real BD-rate. Results show that the proposed model is accurate enough and can be used to perform the optimization described, thereby providing a solution that allows for BD-rate saving up to $7 \%$ or $14 \%$ compared to a static solution where tile sizes are fixed independent on the sequence used.

\section{REFERENCES}

[1] ClassX, Stanford University. http://classx.stanford.edu/. Accessed 18 December 2015.

[2] R. van Brandenburg, O. Niamult, M. Prins, H. Stokking, "Spatial Segmentation For Immersive Media Delivery", 2011.

[3] Christian Weissig, Oliver Schreer, Peter Eisert, Peter Kauff, "The Ultimate Immersive Experience: Panoramic 3D Video Acquisition", Advances in Multimedia Modeling, Lecture Notes in Computer Science Volume 7131, 2012, pp 671-681.

[4] https://www.digitalconcerthall.com/. Accessed Mach 102017

[5] http://www.oculus.com/rift/. Accessed Mach 102017.

[6] http://www.samsung.com/global/microsite/gearvr/. . Accessed Mach 10 2017.

[7] https://www.google.com/get/cardboard/. Accessed Mach 102017 
[8] C. Grünheit, A. Smolic, and T. Wiegand:"Efficient Representation and Interactive Streaming of High-Resolution Panoramic Views", IEEE International Conference on Image Processing (ICIP'02), Rochester, NY, USA, September 2002.

[9] ISO/IEC 23009-1:2014/Amd 2:2015, "Spatial relationship description, generalized URL parameters and other extensions"

[10] A. Mavlankar, B. Girod, "Spatial-Random-Access-Enabled Video Coding for Interactive Virtual Pan/Tilt/Zoom Functionality", IEEE Transactions on Circuits and Systems for Video Technology (CSVT), Vol. 21, No. 5, May 2011.

[11] J. Le Feuvre, C. Concolato, "Tiled-based Adaptive Streaming using MPEG-DASH", ACM Multimedia Systems 2016, May 2016, Austria.

[12] C Lottermann, A Machado, D Schroeder, Y Peng, E Steinbach, "Bit rate estimation for H. 264/AVC video encoding based on temporal and spatial activities", 2014 IEEE International Conference on Image Processing (ICIP)

[13] Y. Liu, Z.G. Li, and Y.C. Soh, "A novel rate control scheme for low delay video communication of H.264/AVC standard," IEEE Transactions on Circuits and Systems for Video Technol- ogy, vol. 17, no. 1, pp. 68-78, 2007 